\title{
Effect of surgical procedures on prostate tumor gene expression profiles
}

\author{
Jie Li ${ }^{1, *}$, Zhi-Hong Zhang ${ }^{2, *}$, Chang-Jun Yin ${ }^{1}$, Christian Pavlovich ${ }^{3}$, Jun Luo ${ }^{3}$, Robert Getzenberg ${ }^{3}$ \\ and Wei Zhang ${ }^{1}$
}

Current surgical treatment of prostate cancer is typically accomplished by either open radical prostatectomy (ORP) or robotic-assisted laparoscopic radical prostatectomy (RALRP). Intra-operative procedural differences between the two surgical approaches may alter the molecular composition of resected surgical specimens, which are indispensable for molecular analysis and biomarker evaluation. The objective of this study is to investigate the effect of different surgical procedures on RNA quality and genome-wide expression signature. RNA integrity number (RIN) values were compared between total RNA samples extracted from consecutive LRP $(n=11)$ and ORP ( $n=24)$ prostate specimens. Expression profiling was performed using the Agilent human whole-genome expression microarrays. Expression differences by surgical type were analyzed by Volcano plot analysis and gene ontology analysis. Quantitative reverse transcription (RT)-PCR was used for expression validation in an independent set of LRP $(n=8)$ and ORP $(n=8)$ samples. The LRP procedure did not compromise RNA integrity. Differential gene expression by surgery types was limited to a small subset of genes, the number of which was smaller than that expected by chance. Unexpectedly, this small subset of differentially expressed genes was enriched for those encoding transcription factors, oxygen transporters and other previously reported surgery-induced stress-response genes, and demonstrated unidirectional reduction in LRP specimens in comparison to ORP specimens. The effect of the LRP procedure on RNA quality and genome-wide transcript levels is negligible, supporting the suitability of LRP surgical specimens for routine molecular analysis. Blunted in vivo stress response in LRP specimens, likely mediated by $\mathrm{CO}_{2}$ insufflation but not by longer ischemia time, is manifested in the reduced expression of stress-response genes in these specimens.

Asian Journal of Andrology (2012) 14, 708-714; doi:10.1038/aja.2012.54; published online 6 August 2012

Keywords: $\mathrm{CO}_{2}$ insufflation; expression microarray; laparoscopic radical prostatectomy; open radical prostatectomy; prostate cancer; stress response

\section{INTRODUCTION}

Currently, for men with localized prostate cancer, surgical resection of the prostatic tumor is most often accomplished by one of two surgical approaches, open radical prostatectomy (ORP) or robot-assisted laparoscopic radical prostatectomy (RALRP), with the latter operation being increasingly practiced in developed countries. In the United States, RALRP accounts for an estimated $80 \%$ of RP surgeries. ${ }^{1}$ In China, while RALRP is rarely performed, a substantial percentage of RP surgeries are nevertheless performed minimally invasively, via traditional laparoscopic radical prostatectomy (LRP). LRP is quite similar to RALRP and involves similar surgical approaches and steps that are different from conventional ORP. ${ }^{2}$ The principal distinctions in sequential surgical steps between LRP and ORP are illustrated in Figure 1. During LRP, the prostate is typically devascularized earlier by severing the vascular pedicles, and is then left in an endobag in the abdomen following surgical resection for about $30 \mathrm{~min}$ until the completion of vesicourethral anastomosis. These procedural differences (Figure 1) may subject an LRP specimen to longer warm ischemia time at body temperature than an ORP specimen. In addition, all LRP surgeries are performed under $\mathrm{CO}_{2}$ insufflation at approximately $15 \mathrm{mmHg}$ pressure.

Dash et al. ${ }^{3}$ showed that postoperative warm ischemia time dramatically increased the expression of a small subset of hypoxia- and stressresponsive genes in ORP specimens. These genes included early growth response 1 (EGR1), jun B proto-oncogene (JUNB), jun D proto-oncogene (JUND) and activating transcription factor 3 (ATF3). ${ }^{3}$ Lin et al. ${ }^{4}$ compared expression levels of 5753 genes in paired prostate biopsies taken immediately following anesthesia (in situ biopsy) and after surgical resection (ex vivo biopsy) during ORP, and identified 62 genes with increased expression levels, but no genes with decreased expression levels, in ex vivo biopsies. These 'surgeryinduced' genes included canonical hypoxia- and stress-responsive genes such as JUNB, JUND, dual specificity phosphatase 1 (DUSP1), immediate early response 2 (IER2) and X-box binding protein 1 (XBP1). More recently, Schlomm et al..$^{5}$ analyzed expression levels of 91 genes during different intra-operative time points of ORP, and identified eight differentially expressed genes, all increased, during the

\footnotetext{
${ }^{1}$ Department of Urology, The First Affiliated Hospital of Nanjing Medical University, Nanjing 210029, China; ${ }^{2}$ Department of Pathology, The First Affiliated Hospital of Nanjing Medical University, Nanjing 210029, China and ${ }^{3}$ Department of Urology, The Johns Hopkins University School of Medicine, Baltimore, MD 21287, USA

* These two authors contributed equally to the study.

Correspondence: Dr W Zhang (zhangwei@njmu.edu.cn)

Received: 18 September 2011; Revised: 15 November 2011; Accepted: 20 April 2012; Published online: 6 August 2012
} 


\begin{tabular}{|ll|}
\hline ORP & $\underline{\text { LRP }}$ \\
Anesthesia & Anesthesia \\
Ligation of dorsal vein & Ligation of dorsal vein \\
Cut dorsal vein \& urethra & Cut bladder neck \\
Retrograde dissection & Cut vascular pedicles \\
Cut vascular pedicles & Antegrade dissection \\
Cut bladder neck & Cut dorsal vein \& urethra \\
Prostate out of body cavity & Anastromosis \\
Anastromosis & Prostate out of body cavity \\
&
\end{tabular}

Figure 1 Summary of procedural differences between ORP and LRP. Tissue ischemia probably occurs earlier in LRP cases than in ORP cases as the vascular pedicles are cut approximately half an hour earlier in LRP than in ORP. In addition, the resected LRP specimen stays in the abdomen for about half an hour unti completion of the vesicourethral anastomosis, while the ORP specimen is processed before the anastomosis is made. Once extracorporeal, the specimens were transported to a pathologist within $10 \mathrm{~min}$ and uniformly processed according to established procedures. LRP, laparoscopic radical prostatectomy; ORP, open radical prostatectomy.

early intra-operative period. In this study, differentially expressed genes also included stress-response genes such as JUNB, DUSP 1 and EGR1. Taken together, these studies suggest that 'surgery-induced' gene expression changes, a potential artifact in expression analysis, are limited to a subset of stress-response gene transcripts that are increased early during the surgical process and further elevated during later intra-operative and post-operative time points.

Surgical prostate tissue specimens are indispensable resources in the evaluation of tissue-based biomarkers and for prostate cancer research. Molecular profiles of the surgical specimens should accurately reflect the inherent tissue biology rather than artifacts of specimen procurement and processing. Since various intra-operative and post-operative variables introduce potential biases that may affect the molecular composition of surgical specimens, the need to identify and correct these biases has been recently emphasized. ${ }^{6,7}$ However, despite the differences in tissue ischemia time and $\mathrm{CO}_{2}$ insufflation between LRP and ORP, few studies have addressed the differential effect of LRP and ORP on RNA quality ${ }^{8}$ and gene expression. ${ }^{9}$ Importantly, a comparison of gene expression profiles from LRP and ORP specimens has not been performed. As a result, the suitability of LRP specimens for gene expression analysis has not been established, and the nature and extent of expression differences influenced by the surgical types are unknown.

In this study, we analyzed consecutive RP specimens procured from either LRP or ORP surgeries and processed uniformly following completion of the surgeries. The goal of this study is to evaluate the effect of the two main prostate cancer surgical types on RNA quality and genome-wide expression profiles.

\section{METHODS AND MATERIALS}

\section{Prostate specimen acquisition and processing}

Participating subjects were consecutive patients undergoing either LRP or ORP for prostate cancer at the First Affiliated Hospital of Nanjing Medical University, China. The study was approved by the Institutional Review Board. Consents were obtained from the study subjects to use excess surgical tissues for molecular analysis.
Once extracorporeal, surgical specimens were transported on ice to a pathologist (ZHZ) within $10 \mathrm{~min}$, and processed for routine pathological evaluation and frozen tissue harvesting strictly according to a tissue-harvesting procedure established at the Johns Hopkins Hospital (USA). ${ }^{10}$ Tissue specimens utilized in this study represent cases qualifying for frozen tissue harvesting from December 2007 to June 2009. Identical specimen handling and tissue-harvesting procedures were applied to LRP and ORP specimens. A serial number was assigned to each harvested specimen, which was stored as two separate optimal cutting temperature compound-embedded blocks at $-80{ }^{\circ} \mathrm{C}$. Prior to RNA extraction, cryosections were prepared from the frozen blocks following pathological examination and block trimming to enrich the tumor content, if necessary. Samples were not allowed to thaw at anytime prior to RNA extraction. Total RNA was extracted from 35 specimens (from 35 cases) using a method described previously ${ }^{11}$ by a blinded technician and transported on dry ice to Shanghai Clinical Research Center (Shanghai, China) for RNA analysis and gene expression profiling. For quantitative reverse transcription (QRT)-PCR validation studies, an independent set of LRP $(n=8)$ and ORP $(n=8)$ cases, harvested during the period between March 2010 and December 2010, were similarly processed for RNA extraction.

\section{RNA expression microarrays}

RNA quality was assessed using the Agilent 2100 Bioanalyzer (Agilent Technologies, Santa Clara, CA, USA). An RNA integrity number was assigned to each of the 35 samples. Gene expression profiling was performed strictly according to the guidelines provided by the Agilent Whole-Genome Expression Microarray system (Agilent Technologies). High-quality genome-wide expression data were generated from 26 RNA samples, including samples from 8 LRP cases, 17 ORP cases and 1 reference RNA sample prepared from a transurethral resection of the prostate (TURP) specimen of benign prostatic hyperplasia (BPH). We used the routine two-color design as described previously. ${ }^{11}$ Briefly, each of the 26 RNA samples, including the TURP-BPH reference sample, was amplified once and labeled with Cy3, and cohybridized with the TURP-BPH reference sample that was similarly amplified but labeled with Cy5. For each sample, expression ratios of $\mathrm{Cy} 3 / \mathrm{Cy} 5$ for 44000 genes/probes constituted the gene expression profile. Microarray experiments for all 26 samples passed strict quality control measures recommended by Agilent Technologies.

\section{Gene expression analysis}

Gene expression ratios for each sample were normalized independently, using the standard locally weighted least squares regression procedure. Locally weighted least squares regression normalization applies a smoothing adjustment that removes intensity-dependent variation, thus balancing the distribution of expression ratios in each sample. Briefly, a smoothing curve was fit to a data set within a sliding window corresponding to $20 \%$ of the total number of genes/probes, following computation of the locally weighted linear regression. To exclude unreliable data points, a quality control filter was applied to all genes/probes, generating a list of 15179 genes/probes meeting the criteria of 'expression values of at least 1000 in at least four samples'. To determine the expression differences between LRP and ORP cases, we performed a Volcano plot analysis using the GeneSpring software (Agilent Technologies). Volcano plot analysis selects genes based on absolute fold change 
(FC) value in combination with $P$ values. For $P$ value calculation, each gene/probe was first assigned an unpaired $t$ score based on comparison of LRP and ORP. Sample labels (LRP or ORP) were then randomly permutated 10000 times to generate empirical distribution of unpaired $t$ test scores for each gene. For each gene/probe, the fraction of permutations with test scores greater than the score from the actual data was defined as the $P$ value. In gene ontology (GO) analysis, the goal is to identify GO terms with significant enrichment of genes in the differentially expressed gene list. We corrected it for multiple testing in GO analysis, and only GO terms with adjusted $P$ values less than 0.05 were considered to be significant.

\section{Q-RT-PCR validation}

For the independent set of RNA samples extracted from LRP $(n=8)$ and ORP $(n=8)$, we carried out standard Q-RT-PCR analysis of three genes, FOSB (forward primer: 5'-GCAACCCACCCTCATCTCTTCC-3'; reverse primer: 5'-CGCCACTGCTGTAGCCACTCAT-3'), JUN (forward primer: 5'-ATGGCTACAGTAACCCCAAGAT-3'; reverse primer: 5'-GAGGTGAGGAGGTCCGAGTTCT-3') and ATF3 (forward primer: 5'-CTCTGCCACCGGATGTCCTCTG-3'; reverse primer: 5'-TCTTTCTCGTCGCCTCTTTTTC-3'), with GAPDH as the control. Briefly, PCR primers were synthesized by GeneRay Biotech, Shanghai, China. First-strand cDNAs were synthesized by PrimeScript one-step RT-PCR kit (Takara, Dalian, China). Real-time RT-PCR was performed using the SYBR Green Premix kit (Takara) in 384-well plates on a 7900 HT Fast Real-Time PCR System (Applied Biosystems, Foster City, CA, USA). Averaged threshold cycle numbers from reactions run in triplicate for each gene in each of the 16 samples were used for comparative threshold analysis by normalizing to GAPDH. The Mann-Whitney $U$ test was used to evaluate expression differences between LRP $(n=8)$ and OPR $(n=8)$ samples. Expression differences were considered significant under $P<0.05$ (one-sided test).

\section{RESULTS}

\section{Clinical and pathological characteristics}

The study involved 35 patients treated for prostate cancer either by LRP $(n=11)$ or ORP $(n=24)$ consecutively at a single institution. The median age at the time of surgery was 69 years old. The majority of patients $(n=19)$ had urinary symptoms at the time of diagnosis. The median prostate-specific antigen (PSA) prior to surgery was $20.7 \mathrm{ng} \mathrm{ml}^{-1}$. The clinical stages of all patients $(n=35)$ were at or above T2a. Gleason scores ranged from 6 to 9, and tumor volumes reached at least $1 \mathrm{~cm}^{3}$ in all cases. Following pathological processing and RNA quality assessment, a subset of harvested specimens did not contain sufficient tumor $(80 \%)$ or did pass quality control (RNA integrity number $(\mathrm{RIN})<6$ ). These specimens

Table 1 Main clinical and pathological variables for the profiled cases for gene expression differences

\begin{tabular}{llll}
\hline & $\operatorname{LRP}(\mathrm{n}=8)$ & ORP $(\mathrm{n}=17)$ & P values \\
\hline Age: median (range), year & $63(30-75)$ & $70(51-75)$ & $>0.05$ \\
PSA: mean (s.d.), ng ml ${ }^{-1}$ & $33.4(46.1)$ & $16.3(10.8)$ & $>0.05$ \\
Gleason score number of cases: & & & $>0.05$ \\
$\quad \leqslant 7$ & 7 & 12 & \\
$\quad 1$ & 1 & 5 & $>0.05$ \\
Total surgery time: mean (s.d.), min & $209(64)$ & $173(41)$ & $>0.05$ \\
Estimated blood loss: mean (s.d.), ml & $838(800)$ & $1176(588)$ & \\
\hline
\end{tabular}

Abbreviations: LRP, laparoscopic radical prostatectomy; ORP, open radical prostatectomy; PSA, prostate-specific antigen. $(n=10)$ were included in RNA quality assessment but excluded in expression studies. Among the cases analyzed for gene expression differences between LRP $(n=8)$ and ORP $(n=17)$, there were no significant differences in PSA, age, Gleason, stage, surgery duration and estimated volume of blood loss (Table 1).

\section{RNA quality comparison}

Total RNA samples prepared from cryosections of fresh frozen LRP $(n=11)$ and ORP $(n=24)$ specimens were subjected to analysis using the Agilent Bioanalyzer. Figure $\mathbf{2} \mathbf{a}$ is a representative gel image of the RNA from three LRP and nine ORP cases. The mean RIN for the LRP specimens $(n=11)$ was 7.6 , while the mean RIN for the ORP specimens was $8.1(n=24)$ (Figure 2b). RIN distribution was not significantly different between LRP and ORP cases $(P=0.915$; Mann-Whitney $U$ test). All RNA samples used in validation studies demonstrated acceptable quality upon examination by regular gel electrophoresis but were not evaluated using the RIN method (data not shown).

\section{Gene expression comparison}

To minimize the contribution of differential epithelium/stroma content to gene expression profiles, we focused on the comparison of LRP $(n=8)$ and ORP $(n=17)$ specimens with at least $80 \%$ tumor content. A $P$ value was assigned to each of the 15179 genes/probes following random permutation analysis comparing LRP and ORP cases. Notably, the numbers of 'significant' genes under each $P$ value category (row marked with FC all, Table 2) were smaller than those expected by chance (data not shown), suggesting an overall small difference in global gene expression between LRP and ORP specimens. Under this scenario, no differentially expressed genes between ORP and LRP cases would be detected using the more stringent false-discovery rates. To overcome this limitation, we used
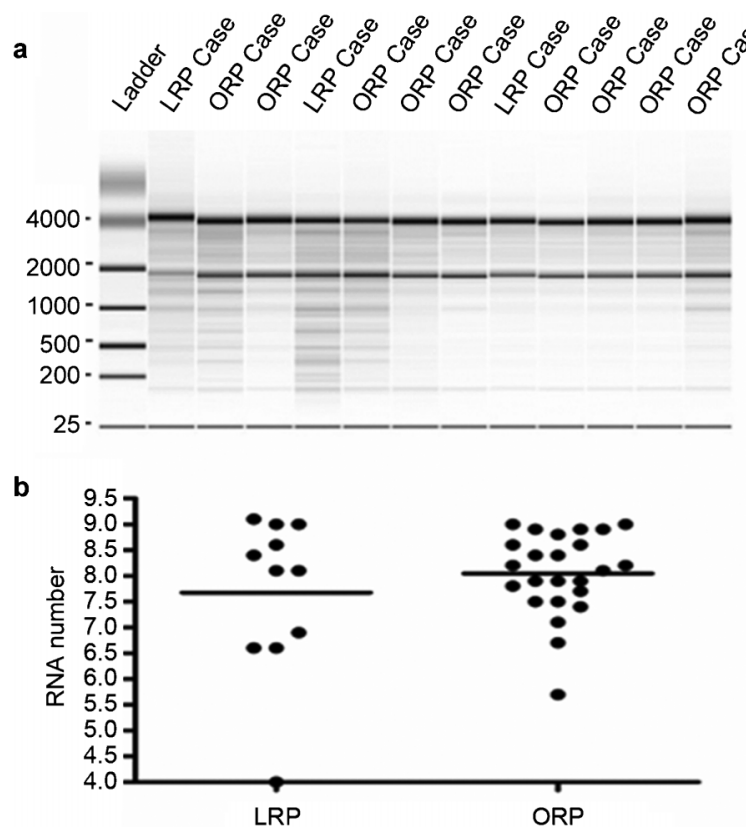

Figure 2 Effect of surgical types on RNA quality. (a) Representative electrophoregram of total RNA extracted from three LRP and nine ORP specimens. The RNA ladder corresponds to size markers as indicated (in bp). Slight shift of the 28-and 18-s bands in the first sample was due to occasional technical errors of the Agilent Bioanalyzer. (b) Comparison of RIN distribution for total RNA extracted from 11 LRP and 24 OPR specimens. LRP, laparoscopic radical prostatectomy; ORP, open radical prostatectomy; RIN, RNA integrity number. 
Table 2 Number of genes/probes under different FC and $\boldsymbol{P}$ values cutoffs

\begin{tabular}{lllllll}
\hline & $\mathrm{P}$ all & $\mathrm{P}<0.05$ & $\mathrm{P}<0.02$ & $\mathrm{P}<0.01$ & $\mathrm{P}<0.005$ & $\mathrm{P}<0.001$ \\
\hline $\mathrm{FC}$ all & $15179(100 \%)$ & $461(3.04 \%)$ & $117(0.77 \%)$ & $44(0.29 \%)$ & $23(0.15 \%)$ & $5(0.03 \%)$ \\
$\mathrm{FC}>1.5$ & $895(5.90 \%)$ & $189(1.25 \%)$ & $61(0.40 \%)$ & $27(0.18 \%)$ & $15(0.10 \%)$ & $5(0.03 \%)$ \\
$\mathrm{FC}>2$ & $225(1.48 \%)$ & $75(0.49 \%)$ & $32(0.21 \%)$ & $18(0.12 \%)$ & $12(0.08 \%)$ & $5(0.03 \%)$ \\
$\mathrm{FC}>3$ & $23(0.15 \%)$ & $17(0.11 \%)$ & $7(0.05 \%)$ & $5(0.03 \%)$ & $3(0.02 \%)$ & $1(0.01 \%)$ \\
\hline
\end{tabular}

Abbreviation: FC, fold change.

Volcano plot analysis, in which both FC and empirical $P$ values were considered for gene identification. Using a cutoff of twofold expression difference and $P<0.05,75$ genes/probes were differentially expressed between ORP and LRP cases (Table 2). This gene/ probe set represented a very small fraction $(0.49 \%)$ of total genes/ probes $(n=15179)$ assessed. The 75 genes/probes were ranked according to $P$ values in Figure 3 a.

Top-ranked genes in Figure 3a, including FOSB, IER2, JUN, $J U N B, A T F 3$ and FOS, are known to be involved in acute stress response and many have been shown to be induced by surgical manipulation. ${ }^{3-5}$ Surprisingly, all of these genes demonstrated lower expression in LRP cases (i.e., upregulated in ORP cases). The 75-gene signature shown in Figure 3a demonstrated the same direction of gene expression change, with 67 (89\%) demonstrating higher expression in ORP cases. House-keeping genes commonly used as control genes in quantitative RT-PCR analysis did not show differential expression (Figure 3b). These house-keeping genes included GAPDH, HPRT1 and TFRC, which were previously used as controls for evaluating expression differences caused by surgical manipulation, ${ }^{5}$ as well as SF3A3, an established control gene for QRT-PCR. ${ }^{12}$

\section{Gene oncology (GO) analysis}

GO analysis of the 75 genes presented in Figure 3a identified two molecular functions, transcription factor activity (GO: 0003700 0000130) and oxygen transport (GO: 0015671), which were significantly enriched (adjusted $P<0.05$ ) in the 75 -gene signature (Figure $4 \mathrm{a}$ and $\mathbf{b}$ ). Notably, all of the 11 transcription factors enriched in the 'transcriptional factor activity' GO term have well-documented roles in mediating inflammatory or stress response in the literature. ${ }^{13-18}$ Three hemoglobin genes $(H B A 2, H B B$ and $H B D)$ were enriched in the 'oxygen transport' GO term (Figure $4 \mathbf{b}$ ). All (100\%) differentially expressed genes enriched in these two GO terms were underexpressed in LRP cases when compared to ORP cases (i.e., upregulated in ORP cases) (Figure $4 \mathrm{a}$ and $\mathbf{4 b}$ ).

\section{Cross-confirmation of genes induced by surgical manipulation}

The typically longer ischemia time associated with LRP specimens would predict an opposite direction of gene regulation, i.e., relative higher expression of stress-response genes in LRP cases. In the study by Lin et al., ${ }^{4}$ surgical manipulation resulted in upregulation of 62 genes but no gene downregulation. To confirm the direction and extent of regulation of these genes, we examined top-ranked surgery-induced genes reported by Lin et al. ${ }^{4}$ in our data set. Among the top 10 genes that matched our gene list, all but one showed upregulation in ORP cases (Figure 4c). These genes included canonical stress-response genes such as DUSP1, IER2, $J U N$ and JUNB that were consistently induced by surgical manipulation and warm ischemia. ${ }^{3-5}$ Therefore, surgery-induced stressresponse gene upregulation was unexpectedly attenuated in LRP specimens.

\section{Validation in independent samples}

To validate the expression differences for stress-response genes, we performed real-time RT-PCR analysis for FOSB, JUN and ATF3 in an independent set of RNA samples extracted from LRP $(n=8)$ and ORP cases $(n=8)$. With the exception of a few cases (e.g., LRP4 and $L R P 7)$, expression levels followed the general trend of lower expression in LRP cases (Figure 5) similar to the pattern observed in expression microarray analysis (Figures 3 and 4). However, the differences between LRP $(n=8)$ and ORP $(n=8)$ cases were not statistically significant ( $P$ values ranged from 0.08 to 0.27 ), possibly due to small sample size. Although unknown variables may account for the outlier cases (e.g., LRP4 and LRP7) that did not follow the general trend, we did not identify such variables among the surgical and pathological parameters recorded for these cases (data not shown).

\section{DISCUSSION}

In this study, we compared the two primary types of prostate cancer surgery in relation to their effects on tissue RNA quality and genome-wide expression profiles. We demonstrated that the LRP procedure does not compromise RNA quality, and that the expression alterations between LRP and ORP are limited to a small subset of stress-response genes, supporting the suitability of LRP specimens for gene expression analysis. Unexpectedly, this small subset of stress-response genes, including previously validated 'surgeryinduced' genes, ${ }^{3-5}$ demonstrated consistently lower expression levels in LRP specimens, a direction of expression change opposite to what would be predicted of specimens with longer ischemia time. We attributed this attenuated stress response in LRP specimens to the effect of $\mathrm{CO}_{2}$ insufflation, raising a tantalizing possibility that an LRP specimen may be less prone to 'surgery-induced' expression artifacts.

Since RNA integrity is a major concern in gene expression studies and may be compromised by longer ischemia times (at body temperature) typically associated with laparoscopic surgeries, the primary goal of the study was to determine whether prostate tumor specimens from LRP are suitable for gene expression analysis. We found that RNA extracted from laparoscopic prostate tumor specimens maintained high quality that was comparable with those from open surgeries. In addition, although the two surgery types did result in altered gene transcript levels (to be discussed in detail below), the effect was limited to a small subset of stress-response genes. In a few previous studies that did not involve expression profiling, the quality of total RNA extracted from limited number of samples was assessed and determined to be adequate for expression analysis. ${ }^{8,9}$ Our study provided reassuring evidence supporting the feasibility of collecting prostate specimens from laparoscopic surgeries for routine molecular analysis. Underscoring the importance of this finding, RALRP will likely become a major source of prostate tumor specimens in the future. Because the surgical procedure of RALRP is quite similar to traditional 
a

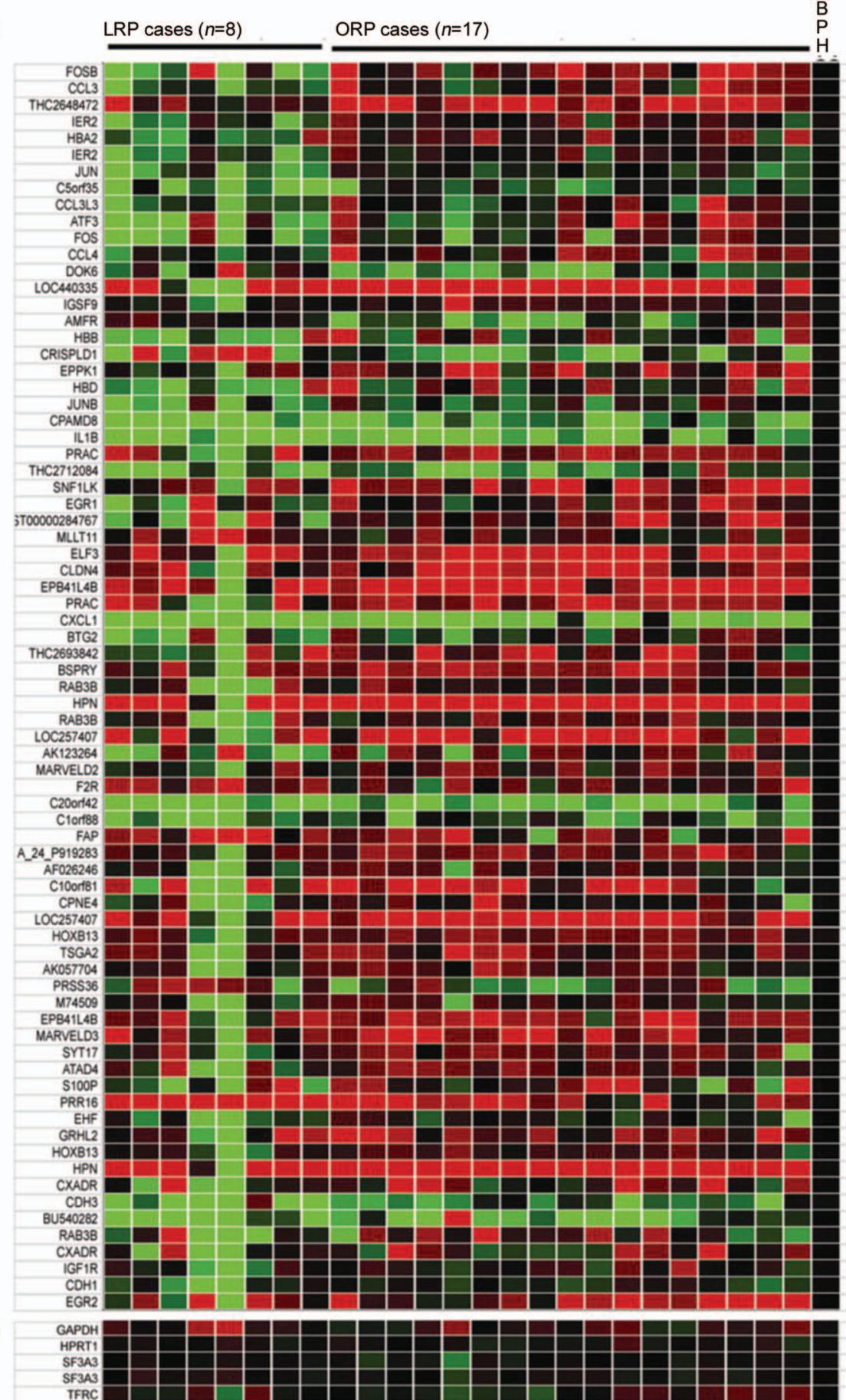

\begin{tabular}{|c|c|c|}
\hline$P$ value & FC & ORP/LRP \\
\hline 0.000 & 4.517 & up \\
\hline 0.000 & 2.742 & up \\
\hline 0.001 & 2.118 & up \\
\hline 0.001 & 2238 & up \\
\hline 0.001 & 2256 & up \\
\hline 0.001 & 2.147 & up \\
\hline 0.002 & 2219 & up \\
\hline 0.003 & 2097 & up \\
\hline 0.003 & 2.239 & up \\
\hline 0.003 & 3.342 & up \\
\hline 0.004 & 3.281 & up \\
\hline 0.004 & 2.153 & up \\
\hline 0.005 & 2254 & down \\
\hline 0.005 & 5.292 & up \\
\hline 0.007 & 2.076 & up \\
\hline 0.007 & 2.116 & down \\
\hline 0.008 & 2.478 & up \\
\hline 0.009 & 3.346 & down \\
\hline 0.011 & 3.192 & up \\
\hline 0.012 & 2309 & up \\
\hline 0.013 & 2.000 & up \\
\hline 0.013 & 2.173 & up \\
\hline 0.014 & 2260 & up \\
\hline 0.014 & 2.044 & up \\
\hline 0.015 & 2220 & up \\
\hline 0.017 & 2.183 & up \\
\hline 0.018 & 2.118 & up \\
\hline 0.018 & 3.847 & up \\
\hline 0.018 & 2.174 & down \\
\hline 0.019 & 2.771 & up \\
\hline 0.020 & 2395 & up \\
\hline 0.020 & 2.516 & up \\
\hline 0.024 & 3.687 & up \\
\hline 0.024 & 2.384 & up \\
\hline 0.024 & 2.038 & up \\
\hline 0.025 & 3.088 & up \\
\hline 0.027 & 2.084 & up \\
\hline 0.028 & 2.710 & up \\
\hline 0.028 & 2.494 & up \\
\hline 0.030 & 2.752 & up \\
\hline 0.031 & 2669 & up \\
\hline 0.031 & 2.190 & up \\
\hline 0.032 & 2063 & up \\
\hline 0.032 & 2.119 & down \\
\hline 0.032 & 2.122 & up \\
\hline 0.034 & 2.029 & up \\
\hline 0.035 & 2333 & down \\
\hline 0.036 & 2.473 & up \\
\hline 0.036 & 2340 & up \\
\hline 0.037 & 3.734 & up \\
\hline 0.037 & 4.084 & up \\
\hline 0.037 & 2.778 & up \\
\hline 0.037 & 2.806 & up \\
\hline 0.038 & 2.462 & up \\
\hline 0.039 & 3.345 & up \\
\hline 0.039 & 2002 & down \\
\hline 0.041 & 2.674 & up \\
\hline 0.041 & 2545 & up \\
\hline 0.042 & 2.097 & up \\
\hline 0.044 & 2.181 & up \\
\hline 0.044 & 3.149 & up \\
\hline 0.045 & 2.705 & up \\
\hline 0.045 & 2.283 & down \\
\hline 0.046 & 2.833 & up \\
\hline 0.046 & 3.108 & up \\
\hline 0.046 & 2.557 & up \\
\hline 0.047 & 2.766 & up \\
\hline 0.047 & 3.163 & up \\
\hline 0.048 & 2.064 & up \\
\hline 0.048 & 2226 & up \\
\hline 0.048 & 2379 & up \\
\hline 0.049 & 3.062 & up \\
\hline 0.050 & 2.208 & up \\
\hline 0.050 & 3.082 & up \\
\hline 0.050 & 2.341 & up \\
\hline 02 & 1.3 & down \\
\hline 0.34 & 1.1 & down \\
\hline 0.23 & 1.1 & down \\
\hline 0.14 & 1.1 & down \\
\hline 0.7 & 1.1 & up \\
\hline
\end{tabular}

Figure 3 Heatmap of expression differences by surgical type. (a) Heatmap of expression ratios for the 75 genes with at least twofold change and $P$ values less than 0.05 following comparison of LRP and ORP specimens. (b) Heatmap of expression ratios for the four commonly used house-keeping genes. All expression ratios are logtransformed ratios of the test sample/BPH. Test samples included eight LRP prostate tumor specimens, $17 \mathrm{ORP}$ prostate tumor specimens and the BPH reference sample itself. The $P$ value, absolute FC and direction of regulation in ORP vs. LRP were provided to the right of the heatmap for each gene/probe. Note that some genes are represented by more than one probe. BPH, benign prostatic hyperplasia; FC, fold change; LRP, laparoscopic radical prostatectomy; ORP, open radical prostatectomy.

non-robotic LRP, and the prostate is handled near-identically, our results foretell the general reliability of molecular data derived from RALRP specimens.

We also found that a small subset of mainly stress-response genes, enriched for those encoding stress-response transcription factors, oxygen transporters and other genes previously shown to be induced by surgical manipulation, were predominantly underexpressed in LRP specimens. Previous studies utilizing smaller microarrays or targeted candidate genes have largely established that stress-response genes were induced early during surgery and further increased gradually as 


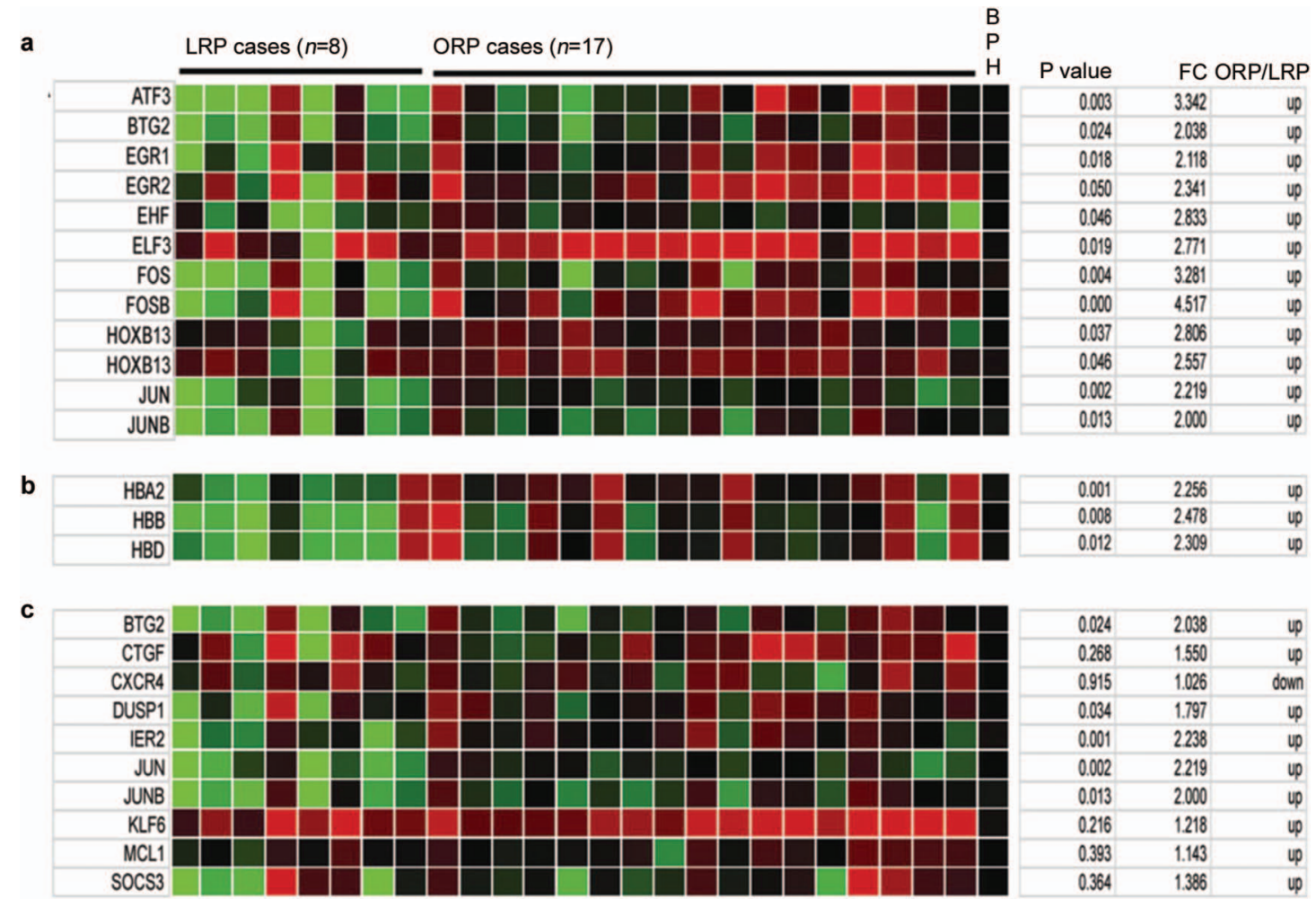

Figure 4 Expression pattern of genes in two GO terms (a, b) and genes that are induced by surgery as reported in Lin et al. ${ }^{4}$ (c). (a) Genes/probes under the GO term 'transcriptional activity'. (b) Genes/probes under the GO term 'oxygen transport'. (c) Genes/probes corresponding to transcripts that are elevated in response to surgery as reported by Lin et al. ${ }^{4}$ As in Figure 3, all expression ratios are log-transformed ratios of the test sample/BPH. Test samples included eight LRP prostate tumor specimens, 17 ORP prostate tumor specimens and the BPH reference sample itself. The $P$ value, absolute FC and direction of regulation in ORP vs. LRP were provided to the right of the heatmap for each gene/probe. Note that some genes are represented by more than one probe. BPH, benign prostatic hyperplasia; FC, fold change; GO, gene ontology; LRP, laparoscopic radical prostatectomy; ORP, open radical prostatectomy.

a function of ischemia time. ${ }^{3-5}$ Given that the LRP specimens are associated with longer ischemia time, one would predict that the same set of genes would be overexpressed in LRP specimens. The surprising finding thus cannot be explained by ischemia time. Another distinction between LRP and ORP is the use of $\mathrm{CO}_{2}$ insufflation during LRP. Hanly et al. ${ }^{19}$ proposed that $\mathrm{CO}_{2}$-mediated attenuation of the acute phase response may be the underlying mechanism of reduced systemic inflammatory response reported in earlier laparoscopic surgeries. ${ }^{20-22}$ The immune-modulatory effect of $\mathrm{CO}_{2}$ is apparently mediated by peritoneal acidosis as acidic solutions had the same effect. ${ }^{23}$ Therefore, we speculate that abdominal $\mathrm{CO}_{2}$ insufflation, rather than reduced tissue trauma, may account for underexpression of stress-response genes in LRP specimens compared with ORP specimens.

If the increased expression of surgery-induced stress-response genes is an artifact, as indicated in previous studies using ORP specimens, ${ }^{3-5}$ $\mathrm{CO}_{2}$ insufflation may have prevented this artifact from occurring in the laparoscopic cases. If this notion holds true, LRP specimens may be more representative of the true state of the tissue biology. The concurrent underexpression of genes encoding transcription factors and oxygen transporters in LRP cases suggests that while prostate tissues would normally respond to ischemia by acute increase of their transcript levels as observed in open cases, $\mathrm{CO}_{2}$ insufflation blunts this response. One likely underlying mechanism is that $\mathrm{CO}_{2}$-induced local acidosis attenuates the surgery-induced stress response in the prostate during LRP. These in vivo results, however, need to be validated using in vitro models.
Our study is currently limited by the number of specimens available for further analysis. The small number of samples may have contributed to the lack of significant genes following correction of multiple testing. Therefore, we mainly relied on a less-stringent method for identification of differentially expressed genes (Figure 3a). However, GO analysis did find two molecular functions (Figure 4a and $4 \mathrm{~b}$ ) that are significantly different even after correction of multiple testing. The cellular origin of the hemoglobin transcripts in the 'oxygen transporter' GO term is not known, though induced expression of hemoglobin has been reported in nonerythroid cells such as macrophages and in the developing prostate. ${ }^{24,25}$ Validation in a small set of independent samples was performed. Though the small sample size may have contributed to the lack of statistically significant differences (Figure 5), we observed the consistent trend of lower expression of stress-induced genes in LRP cases. Further validation will await the availability of more surgical specimens currently being systemically collected following LRP and ORP. A further potential limitation is that the prostate cancer cases described in this study, currently representative of the patient population in China, presented with more locally advanced disease than patients in the United States, possibly due to differences in prostate cancer screening. Further studies are needed to determine whether LRP or RALRP procedures also attenuate the stress response in earlier stage prostate tumors.

\section{CONCLUSIONS}

In summary, laparoscopic procedures alone do not negatively influence prostate tumor RNA quality. Thus, LRP or RALRP specimens 

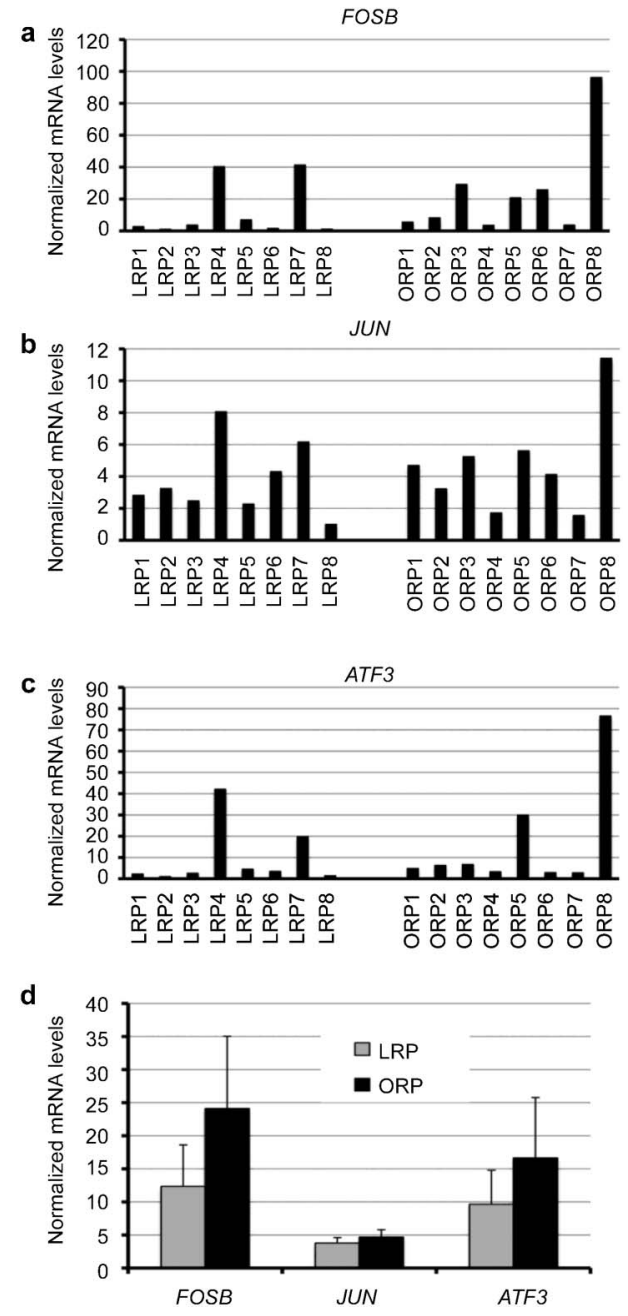

Figure 5 Real-time RT-PCR validation of expression differences between LRP and ORP cases. Expression differences of FOSB (a), JUN (b), ATF3 (c) between $\operatorname{LRP}(n=8)$ and ORP $(n=8)$ cases. d), Expression differences of FOSB, JUN, ATF3. LRP, laparoscopic radical prostatectomy; ORP, open radical prostatectomy.

should prove adequate for routine molecular analysis. The effect of laparoscopic surgery on gene expression is limited to a small subset of mainly stress-response genes that are predominantly underexpressed in laparoscopic cases in comparison to open cases. These stressresponse genes overlap with previously reported surgery-induced genes, suggesting that $\mathrm{CO}_{2}$ insufflation during LRP attenuates the stress response in prostate tissues.

\section{AUTHOR CONTRIBUTIONS}

WZ, RG and CJY conceived and designed the study. Jie L, ZHZ and CJY performed the experiments. Jie L and Jun L performed the statistical analyses. Jie L and ZHZ drafted and revised the manuscript. RG and $\mathrm{CP}$ critically reviewed the manuscript. All authors read and approved the final version.

\section{COMPETING FINANCIAL INTERESTS}

The authors declare that they have no competing financial interests.

\section{ACKNOWLEDGMENTS}

The authors would like to thank Dr Hongjun Yang (Shanghai Clinical Research Center, Shanghai, China) and Dr Huazheng Xiao (Shanghai Biochip Co., Shanghai, China) for assistance and cooperation. The project is funded by grants from National Natural Science Foundation of China (No. 30872575 and No. 30973199).

1 Su LM. Robot-assisted radical prostatectomy: advances since 2005. Curr Opin Urol 2010; 20: 130-5.

2 Schaeffer EM, Loeb S, Walsh PC. The case for open radical prostatectomy. Urol Clin North Am 2010; 37: 49-55.

3 Dash A, Maine IP, Varambally S, Shen R, Chinnaiyan AM et al. Changes in differential gene expression because of warm ischemia time of radical prostatectomy specimens. Am J Pathol 2002; 161: 1743-8.

4 Lin DW, Coleman IM, Hawley S, Huang CY, Dumpit R et al. Influence of surgical manipulation on prostate gene expression: implications for molecular correlates of treatment effects and disease prognosis. J Clin Oncol 2006; 24: 3763-70.

5 Schlomm T, Näkel E, Lübke A, Buness A, Chun FK et al. Marked gene transcript level alterations occur early during radical prostatectomy. Eur Urol 2008; 53: 333-44.

6 Compton CC. The surgical specimen is the personalized part of personalized cancer medicine. Ann Surg Oncol 2009; 16: 2079-80.

7 Febbo PG, Kantoff PW. Noise and bias in microarray analysis of tumor specimens. J Clin Oncol 2006; 24: 3719-21.

8 Best S, Sawers Y, Fu VX, Almassi N, Huang W et al. Integrity of prostatic tissue for molecular analysis after robotic-assisted laparoscopic and open prostatectomy. Urology 2007; 70: 328-32.

9 Ricciardelli C, Bianco-Miotto T, Jindal S, Dodd TJ, Cohen PA et al. Comparative biomarker expression and RNA integrity in biospecimens derived from radical retropubic and robot-assisted laparoscopic prostatectomies. Cancer Epidemiol Biomarkers Prev 2010; 19: 1755-65.

10 Bova GS, Fox WM, Epstein JI. Methods of radical prostatectomy specimen processing: a novel technique for harvesting fresh prostate cancer tissue and review of processing techniques. Mod Pathol 1993; 6: 201-7.

11 Dunn TA, Chen S, Faith DA, Hicks JL, Platz EA et al. A novel role of myosin VI in human prostate cancer. Am J Pathol 2006; 169: 1843-54.

12 Hu R, Dunn TA, Wei S, Isharwal S, Veltri RW et al. Ligand-independent androgen receptor variants derived from splicing of cryptic exons signify hormone-refractory prostate cancer. Cancer Res 2009; 69: 16-22

13 Yan SF, Harja E, Andrassy M, Fujita T, Schmidt AM. Protein kinase C beta/early growth response-1 pathway: a key player in ischemia, atherosclerosis, and restenosis. J Am Coll Cardiol 2006; 48: (9 Suppl 1), A47-55.

14 Wu J, Duan R, Cao H, Field D, Newnham CM et al. Regulation of epithelium-specific Ets-like factors ESE-1 and ESE-3 in airway epithelial cells: potential roles in airway inflammation. Cell Res 2008; 18: 649-63.

15 Stelnicki EJ, Arbeit J, Cass DL, Saner C, Harrison M et al. Modulation of the human homeobox genes PRX-2 and HOXB13 in scarless fetal wounds. J Invest Dermatol 1998; 111: 57-63

16 Savkovic V, Gaiser S, lovanna JL, Bödeker H. The stress response of the exocrine pancreas. Dig Dis 2004; 22: 239-46.

17 Hess J, Angel P, Schorpp-Kistner M. AP-1 subunits: quarrel and harmony among siblings. J Cell Sci 2004; 117(Pt 25): 5965-73.

18 Hai T, Wolfgang CD, Marsee DK, Allen AE, Sivaprasad U. ATF3 and stress responses. Gene Expr 1999; 7: 321-35

19 Hanly EJ, Mendoza-Sagaon M, Murata K, Hardacre JM, de Maio A et al. $\mathrm{CO}_{2}$ pneumoperitoneum modifies the inflammatory response to sepsis. Ann Surg 2003; 237: 343-50.

20 Glaser F, Sannwald GA, Buhr HJ, Kuntz C, Mayer H et al. General stress response to conventional and laparoscopic cholecystectomy. Ann Surg 1995; 221: 372-80.

21 Jakeways MS, Mitchell V, Hashim IA, Chadwick SJ, Shenkin A et al. Metabolic and inflammatory responses after open or laparoscopic cholecystectomy. Br J Surg 1994; 81: 127-31.

22 Redmond HP, Watson RW, Houghton T, Condron C, Watson RG et al. Immune function in patients undergoing open vs laparoscopic cholecystectomy. Arch Surg 1994; 129: 1240-6.

23 Hanly EJ, Aurora AA, Shih SP, Fuentes JM, Marohn MR et al. Peritoneal acidosis mediates immunoprotection in laparoscopic surgery. Surgery 2007; 142: 357-64.

24 Liu L, Zeng M, Stamler JS. Hemoglobin induction in mouse macrophages. Proc Nat Acad Sci USA 1999; 96: 6643-7.

25 Pritchard C, Mecham B, Dumpit R, Coleman I, Bhattacharjee M et al. Conserved gene expression programs integrate mammalian prostate development and tumorigenesis. Cancer Res 2009; 69: 1739-47. 